\title{
Block Adjustment and Image Matching of WorldView-3 Stereo Pairs and Accuracy Evaluation
}

\author{
Zuo Chen ${ }^{1,2, *}$, Xiao Xiao ${ }^{1,2}$, Hou Qingming ${ }^{1,2}$, Li Bing ${ }^{1,2}$ \\ ${ }^{1}$ Beijing Institute of Mapping and Surveying, Beijing, China; \\ ${ }^{2}$ Beijing Key Laboratory of Urban Spatial Information Engineering, Beijng, China; 495631889@qq.com
}

\section{Commission III, ICWG III/IVb}

KEY WORDS: WorldView-3; RPC Block Adjustment Model; DSM; Image Matching

\begin{abstract}
:
WorldView-3, as a high-resolution commercial earth observation satellite, which is launched by Digital Global, provides panchromatic imagery of $0.31 \mathrm{~m}$ resolution. The positioning accuracy is less than 3.5 meter CE90 without ground control, which can use for large scale topographic mapping. This paper presented the block adjustment for WorldView-3 based on RPC model and achieved the accuracy of 1:2000 scale topographic mapping with few control points. On the base of stereo orientation result, this paper applied two kinds of image matching algorithm for DSM extraction: LQM and SGM. Finally, this paper compared the accuracy of the point cloud generated by the two image matching methods with the reference data which was acquired by an airborne laser scanner.

The results showed that the RPC adjustment model of WorldView-3 image with small number of GCPs could satisfy the requirement of Chinese Surveying and Mapping regulations for 1:2000 scale topographic maps. And the point cloud result obtained through WorldView-3 stereo image matching had higher elevation accuracy, the RMS error of elevation for bare ground area is $0.45 \mathrm{~m}$, while for buildings the accuracy can almost reach 1 meter.
\end{abstract}

\section{INTRODUCTION}

In recent years, domestic and foreign surveying and mapping satellites have developed rapidly. Many countries attach great importance to the development and application of surveying satellites and have successively launched different types of surveying satellites. Combining multiple sensor approaches, more flexible imaging mechanisms, and high-precision orbit determination technology, surveying satellites are evolving toward higher spatial resolution, temporal resolution, and spectral resolution.

The United States is in the leading position in the field of surveying satellite technology and has the most number of optical surveying satellites with the highest spatial resolution. Commonly there are Quickbird, IKONOS, Woldview series satellites, GeoEye series satellites, OrbView-3, with spatial resolution up to 0.25 meter and positioning accuracy of 3 meters without ground control point. And these high-resolution satellite images can be used for 1:5000, 1:2000 large-scale topographic mapping. WorldView-3 imagery is the highest resolution product offered by commercial satellite company and its panchromatic band has a spatial resolution of up to 0.31 meters. In addition, the positioning accuracy is less than 3.5 meter CE90 without ground control. In terms of spectrum, 8 shortwave infrared SWIR bands and 12 CAVIS bands for atmospheric correction were added to the 8 multispectral spectral bands of WorldView-2. Despite the superior imaging performance, it also implements rapid satellite attitude adjustment by setting up large torque actuators, and uses a single camera for quick redirection to achieve various imaging modes such as multiple targets imaging in the same orbit, multiple strip imaging of large-area target at one track, and same-track multi-view stereo imaging for one target (LI Guoyuan,2015). The agility of the WorldView-3 imaging mechanism has increased the scope of observations, shortened the revisit period, and improved satellite application capabilities. Previous applications based on stereoscopic satellite imagery have been more common in small-scale applications below 1:5000. For 1:2000 large-scale mapping applications are rare, WorldView-3 stereoscopic imagery provides the possibility for large-scale surveying and mapping applications

\section{DATA AND SURVEY AREA}

The experimental data is one pair of WorldView-3 stereo images of Shijingshan District in Beijing, which was acquired in December 2016. Obtained at same-track multi-angle stereoscopic imaging mode (as shown in Figure 1), the overlap degree of tow images is close to $100 \%$.

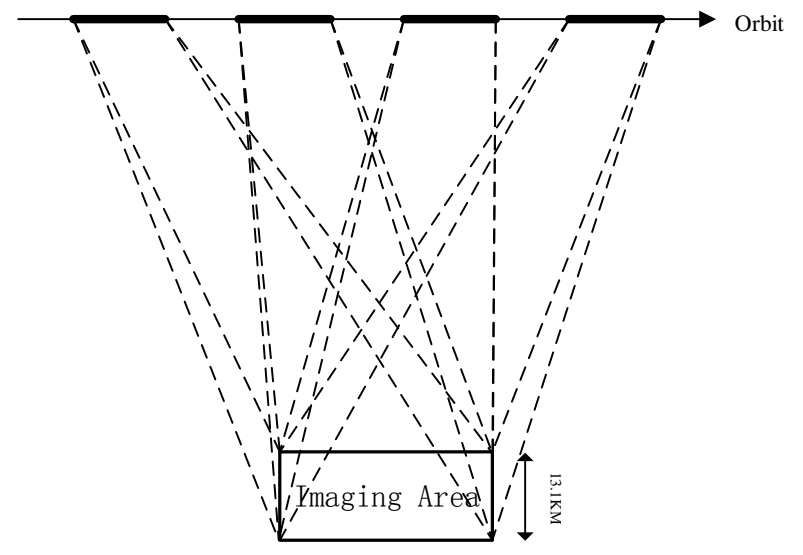

* Corresponding author 
Figure1. Stereo imaging mode

The images cover an area of about 100 square kilometers, and elevation within survey area varies from 30 meters to 600 meters. Yongding River runs through north and south of survey area, and there are relatively dense houses in plain on both sides of the river. The survey area covers various landforms such as houses, roads, rivers and hills.

\section{BLOCK ADJUSTMENT OF WORLDVIEW-3 IMAGE PAIRS AND ACCURACY}

\subsection{Block Adjustment Model}

There are two main methods available for the block adjustment of high-resolution satellite stereo image pairs: physical camera model and Rational Polynomial Coefficient camera model. The physical camera model takes into account various factors such as orbital position, sensor attitude, atmospheric refraction, and surface undulation during satellite imaging, and a complex set model constructed using the physical conditions of imaging. The theory is tight, which can truly reflect the geometric relationship between the image and the object. The RPC model transforms three-dimensional object space coordinates into twodimensional image space coordinates through a rational polynomial function, it provides a simple and accurate means of communicating camera object-image relationship. The block adjustment based on RPC model can achieve the same accuracy as the physical camera model (Grodecki J and Dial G, 2003). The RPC model is as follows.

$$
\begin{aligned}
& x=\frac{p_{1}\left(X_{n}, Y_{n}, Z_{n}\right)}{p_{2}\left(X_{n}, Y_{n}, Z_{n}\right)} \\
& y=\frac{p_{3}\left(X_{n}, Y_{n}, Z_{n}\right)}{p_{4}\left(X_{n}, Y_{n}, Z_{n}\right)}
\end{aligned}
$$

$(\mathrm{x}, \mathrm{y})$ is image-space coordinate, $(X, Y, Z)$ is object-space coordinate. The functional model is in the form of a ratio of two polynomials.

This paper applied the affine transformation in the image space added to RPC model for block adjustment, the math model is defined as follows (Grodecki J and Dial G, 2003):

$$
\begin{aligned}
& x_{k}+a_{0}+a_{1} x_{k}+a_{2} y_{k}=R P C_{x}\left(X_{k}, Y_{k}, Z_{k}\right) \\
& y_{k}+b_{0}+b_{1} x_{k}+b_{2} y_{k}=R P C_{y}\left(X_{k}, Y_{k}, Z_{k}\right)
\end{aligned}
$$

$\left(x_{k}, y_{k}\right)$ is the measured image-space coordinates; $\left(X_{k}, Y_{k}, Z_{k}\right)$ is object-space coordinate; $\left(a_{0}, a_{1}, a_{2}\right),\left(b_{0}, b_{1}, b_{2}\right)$ is affine transform coefficient to capture the discrepancies between the nominal and the measured image-space coordinates.

The image space affine transformation based on RPC model are widely used for block adjustment of high-resolution satellite images. ZhangLi (2009) presented an approach for block adjustment based on RPC model with sparse GCPs by using SPOT-5 satellite images, which results could achieve 5-9 $\mathrm{m}$ in planimetric and 2-3 $\mathrm{m}$ in height direction. Xia Xianli (2010) applied this method to perform 1:5000 mapping experiments on worldview1 images, the DLG results meet the accuracy of 1:5000 topographic maps, providing a new method for border mapping. Zhang Guo (2014) applied RPC model of ZY-3 long strip scenes for block adjustment, when ground control points were deployed around the corners of the survey area, the RMS of the plane and elevation of long strip scenes was $2.5 \mathrm{~m}$ and $1.6 \mathrm{~m}$ respectively.

\subsection{Experimental Results}

40 GCPs as shown in Figure 3 were selected to demonstrate the block adjustment accuracy of WorldView-3 stereo pairs. This paper experimented 4 different kinds of control point layout scheme, which are 1) 4 points around image corner as control points and other 36 points as check points ; 2) 5 points as control points, 4 of them at image corners and the other one at the image center, and other 35 points as check points; 3) 9 points as control points, which are picked evenly in three rows throughout the image and the other 31 points as check points; 4) 12 points as control point, which are picked evenly in four rows throughout the image and the other 28 points as check points. The accuracy of different schemes is shown in Table1.

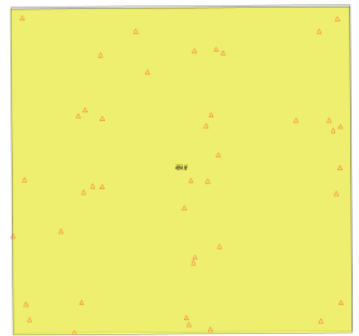

Figure 2. Distribution of control points in the study area

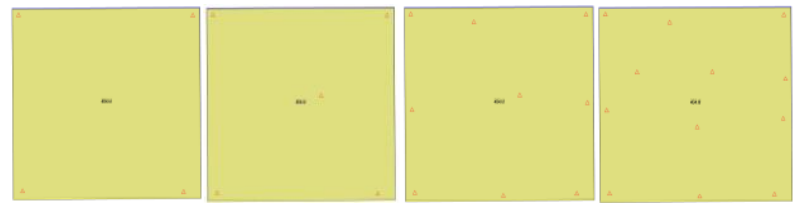

Figure 3. Control point layout schemes

\begin{tabular}{|c|c|c|c|c|c|c|}
\hline & \multicolumn{3}{|c|}{ RMS(m) } & \multicolumn{3}{c|}{ Maximum residual (m) } \\
\cline { 2 - 7 } & $\mathrm{X}$ & $\mathrm{Y}$ & $\mathrm{Z}$ & $\mathrm{X}$ & $\mathrm{Y}$ & $\mathrm{Z}$ \\
\hline $\begin{array}{c}4 \text { in } \\
\text { corner }\end{array}$ & 0.235 & 0.249 & 0.213 & 0.531 & 0.502 & 0.445 \\
\hline $\begin{array}{c}5 \\
\text { points }\end{array}$ & 0.214 & 0.254 & 0.216 & 0.503 & 0.476 & 0.501 \\
\hline $\begin{array}{c}3 \\
\text { rows }\end{array}$ & 0.192 & 0.254 & 0.224 & 0.391 & 0.449 & 0.455 \\
\hline $\begin{array}{c}4 \\
\text { rows }\end{array}$ & 0.193 & 0.237 & 0.216 & 0.433 & 0.458 & 0.471 \\
\hline
\end{tabular}

Table 1. Accuracy of different control point layout

From the orientation results of different layout schemes, it can be seen that the adjustment model with 4 control points around the corners of the image can achieve the accuracy of 0.27 meter in plane and 0.21 meter in elevation, which can satisfy the requirement of Chinese Surveying and Mapping regulations for 1:2000 scale topographic maps. In addition, according to the experiment, the orientation accuracy did not increase with raising amount of control points. 


\section{IMAGE MATCHING AND ACCURACY EVALUATION}

\subsection{Matching Approach and Comparison}

This paper applied two kinds of image matching algorithm, Least Square Matching (LSM) and Semi-Globe Matching (SGM), for DSM extraction.

The LSM uses Normalized Cross Correlation (NCC) to measure the similarity of two points in two images and then the result is affected by window size, which determines the area used for computing the correlation coefficient between the left and right images. For areas containing minimal variation in topographic relief, grey level, or color intensity (such as desert, agricultural fields, grassy areas), use the 9X9 or greater. For areas containing large degrees of topographic relief, grey level variation, and color intensity variation, use a smaller window size, such as $5 \mathrm{X}$ 5. A smaller window size limits the geometric distortion and improves the chance for a match. LSM uses least squares to refine the correlation to provide improved sub-pixel results, so it can improve the matching accuracy. Although LSM could obtain high matching accuracy, it cannot acquire matching points in the area which is lack of features, texture duplication and shadow occlusion, resulting in discontinuity of the point cloud.

Semi-Globe Matching is a dense matching algorithm that uses image mutual information as match measure, it searches correlated points by approximating the minimization of a global energy combining path wise optimizations from all directions through the image and search strategy adopts dynamic programming calculation(Hirschmüller,2008). SGM can acquire dense points which density is comparable to the point cloud data acquired by LiDAR, so that it is a better solution to the discontinuity caused by less gray features such as shadow occlusion. The main flow of the algorithm is as follows:

1) Using mutual information of two images as matching costs. 2) The energy function is defined based on the matching cost. By searching in multiple directions, search for the parallax that minimizes the energy function.

3) Parallax optimization.

This paper uses two methods to obtain point cloud data in the survey area, and compares them from several aspects, such as point cloud density, continuity, and elevation accuracy.

1) Point density comparison. The average point density of the point cloud obtained from the LSM matching is 1 meter 2 points; the average point density of the point cloud obtained from the SGM matching is 1 meter 10 points, and the results are compared as shown in the figure below. Therefore, the point cloud obtained by the SGM method is finer for terrain and ground objects.
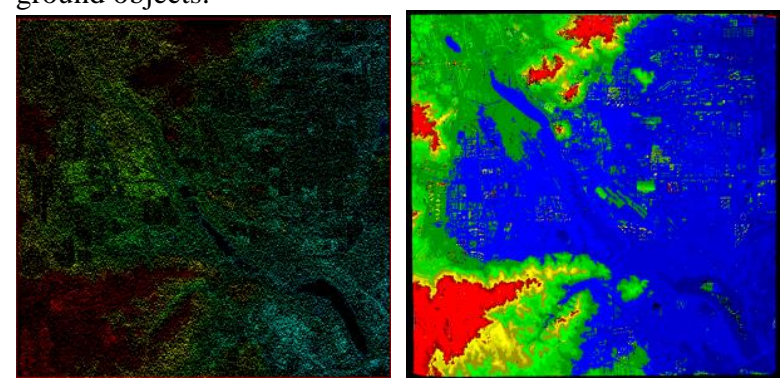

Figure 4. The left shows the LSM result, the right shows the SGM result

2) Point continuity comparison. In the shadowed and weakly textured areas, the point cloud obtained by LSM matching will appear loopholes; but the result obtained by SGM matching are not affected by shadows or lack of information, and continuous point clouds are obtained. In the shadow areas of high-rise buildings, the matching effect is as shown below.
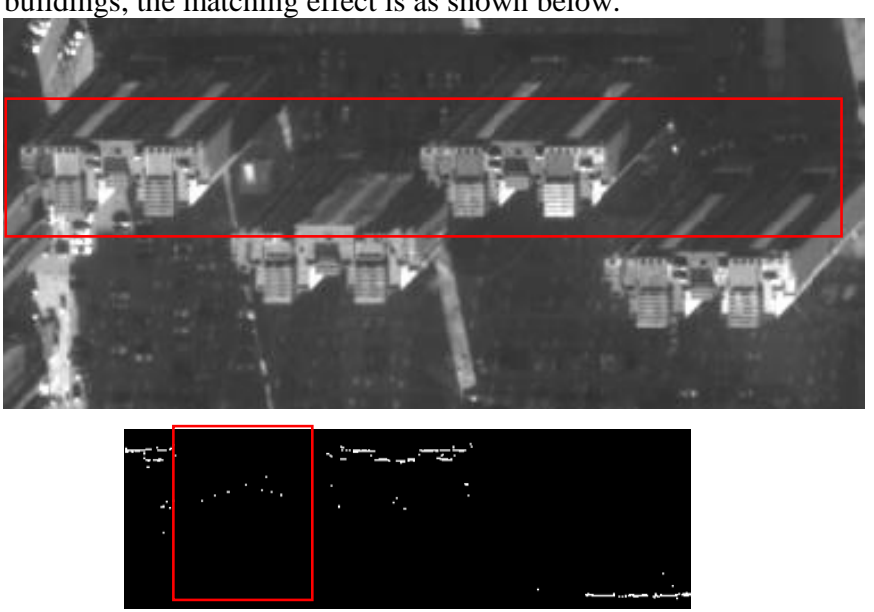

5.a LSM result

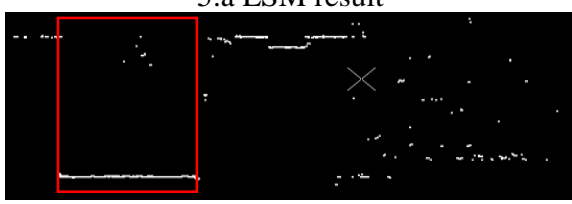

5.b SGM result

Figure 5. Comparison of results at shadows

3) Elevation accuracy comparison. The reference data which was acquired by an airborne laser scanner was used to evaluate the elevation accuracy of two kinds of matching point cloud results. This paper supposed elevation of airborne laser data as ground truth, so the difference between matching point elevation and laser elevation could be used to calculate root mean square error (RMSE). There were 709 ground points involved in the statistic calculation. The elevation residuals of the two matching point cloud results are obtained, and the elevation residual distributions of different levels are compared as shown in the figure below. The red points elevation residual are more than 5 meters; the yellow points elevation residual are between 1 meter and 5 meters and the green points elevation residual are less than 1 meter.

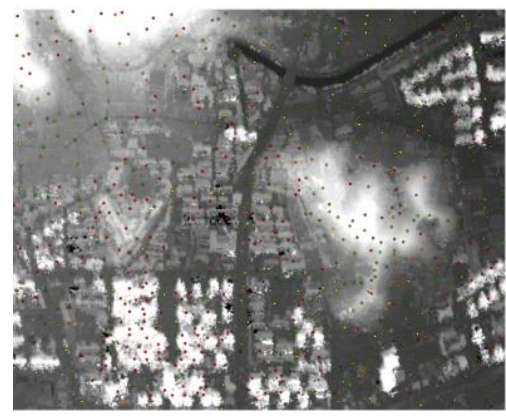

6.a LSM result 


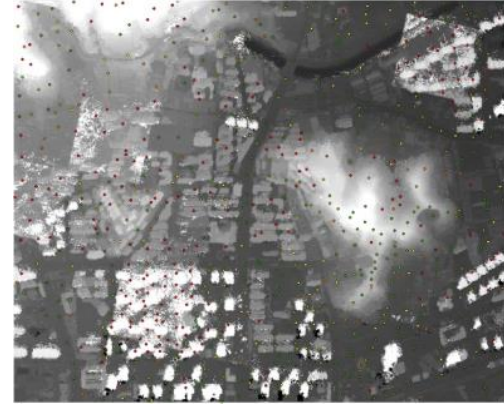

6.b SGM result

Figure 6. Elevation residual distributions of different levels

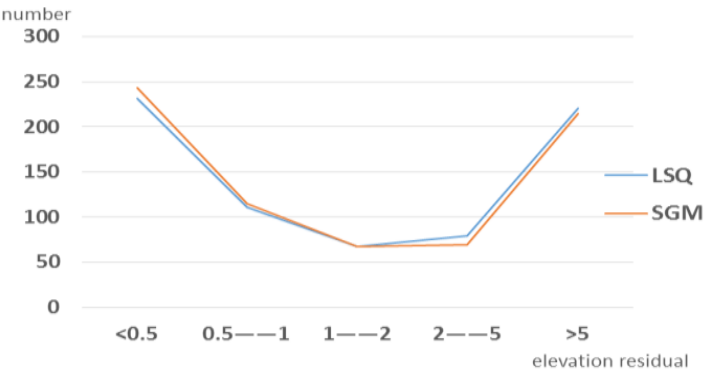

Figure 7. Comparison of the residuals of the two methods

From the results of the regional distribution of residuals, it can be seen that the two methods in the shadowed area of the image will produce false matching points. On the other hand, from the comparison of the number of residuals at different levels, the reliability of SGM matching is slightly higher than that of LSM method.

\subsection{Elevation Accuracy Evaluation}

This paper generated matching point using SGM algorithm, and then compared it with the laser point data in the whole study area. The effect of DSM data generated by two types of point clouds is shown in Figure 9. It can be seen that although the average point density obtained by SGM matching is higher than laser point data, the laser point cloud data is more continuous and uniform and can better reflect the boundary and details of the feature.

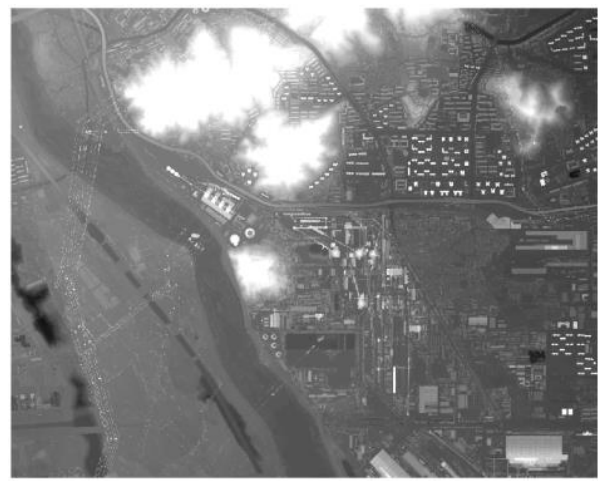

8.a Laser DSM

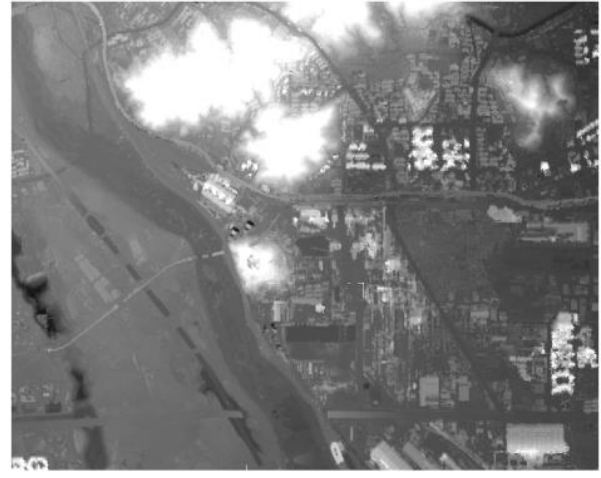

8.b Matching DSM

Figure 8. Comparison of laser DSM and matching DSM

The different was computed by the minus of the interpolated heights from laser DSM and matching DSM. Finally, there were total 2,186 ground check points and 15,768 building check points involved in height difference statistics, excluding change area s shadow area and lack of texture area. The elevation accuracy of our generated DSM for bare ground area is $0.45 \mathrm{~m}$, while for buildings is $1.36 \mathrm{~m}$.

\section{CONCLUSIONS}

This paper applied block adjustment and image matching for WorldView-3 stereo image pairs. Firstly, as demonstrated in this paper, for WorldView-3 image pairs, the RPC adjustment model only with few ground control points can achieve the accuracy of 1:2000 topographic mapping. Secondly, the paper compared the effects and accuracy of two matching methods, namely LSM and SGM. The results showed that SGM could obtain denser and more reliable point cloud results, but it was still affected by image shadows and lack of texture. Through the validation of WorldView-3 matching point cloud elevation accuracy, we can see that WorldView-3 stereo images can achieve higher elevation accuracy. The paper verified the accuracy of WorldView-3 data, confirming that WorldView-3 stereo image data can satisfy large-scale surveying and mapping products with good control. Using WorldView-3 image is a much faster method to obtain $4 \mathrm{D}$ products than traditional aerial photogrammetry approach.

\section{REFERENCES}

Grodecki J, Dial G, J., 2003, Block Adjustment of HighResolusion Satellite Images Described by Rational Polynomials. Photogrammetric Engineering \& Remote Sensing, 69(1), pp. 59-68.

Hirschmuller H, J., 2008. Stereo Processing by Semiglobal Matching and Mutual Information. IEEE Transactions on Pattern Analysis and Machine Intelligence, 30(2). pp. 328-341. Jacobsen K, C. (2007): Orientation of High Resolution Optical Space Imaeges, In: ASPRS 2007 Annual Conference, Tampa, Florida.

Li Zhang, Armin Gruen, J., 2006. Multi-image Matching for DSM Generation from IKONOS Imagery. ISPRS Journal of Photogrammetry \& Remote Sensing, 60(2006), pp. 195-211.

LI Guoyuan, HU Fen, ZHANG Chongyang, GAO Xiaoming, CHEN Chen, J., 2015. Introduction to Imaging Mode of WorldView-3 Satellite Quality Preliminary Evaluation. Bulletin of Surveying and Mapping, pp. 11-16. 
The International Archives of the Photogrammetry, Remote Sensing and Spatial Information Sciences, Volume XLII-3, 2018 ISPRS TC III Mid-term Symposium "Developments, Technologies and Applications in Remote Sensing", 7-10 May, Beijing, China

XIA Xianli, HAN Ming, WEN Guang, ZHAO Junhua, J., 2010.

Experiment of the Production of 1:5000 DLG Based on

WorldView-1 Imagery. Bulletin of Surveying and Mapping,

2010(1). pp. 37-39.

ZHANG Guo, PAN Hongbo, TANG Xinming, LI Deren, J.,

2014. Block Adjustment of ZY-3 Long Strip Scenes. Geomatics and Information Science of Wuhan University , 39(9), pp. 10981102 .

ZHANG Li, ZHANG Jixian, CHEN Xianyang, AN Hong, J., 2009. Block-Adjustment with SPOT-5 Imagery and Sparse GCPs Based on RFM. Acta Geodaetica et Cartographica Sinica, 38(4), pp. 302-310. 